\title{
MODELAGEM PRÉ-COLHEITA DA ESTRUTURA DIAMÉTRICA DE PLANTIOS DE Pinus spp. POR MEIO DE DISTRIBUIÇÕES PROBABILÍSTICAS
}

\author{
Fernando da Silva \\ Eng. Florestal, Mestrando do Instituto Nacional de Pesquisas da Amazônia, Manaus, AM, Brasil - silvaflorestal@ gmail.com
}

Recebido para publicação: 15/10/2010 - Aceito para publicação: 22/12/2011

\begin{abstract}
Resumo
O objetivo foi estudar o comportamento das curvas de distribuição diamétrica de plantios de Pinus spp. no município de Palmas, PR. Os dados são de um povoamento de 87 ha, com 18 anos de idade. As funções probabilísticas Normal, Log-normal, Gamma, Beta, SB de Johnson, Weibull 2P, Weibull 3P e Weber foram ajustadas, utilizando-se para estimativa dos parâmetros o Método dos Momentos, o Método da Máxima Verossimilhança e o Método de Mínimos Quadrados. A qualidade dos ajustes das distribuições foi avaliada por meio do teste de $\chi^{2}$, adotando $5 \%$ de probabilidade e o desvio relativo entre as somas observada e estimada dos diâmetros. Verificou-se a sobreposição das curvas em algumas classes, não sendo possível discriminar qual a melhor distribuição somente observando os histogramas. As curvas foram comparadas analiticamente pelo teste de qui-quadrado $\left(\chi^{2}\right)$, que revelou diferenças significativas entre as curvas observada e estimada. Procedeu-se à análise dos desvios relativos entre as somas observada e estimada dos diâmetros, apenas para as funções que se mostraram aderentes no teste de $\chi^{2}$. Dentre as funções testadas, Weibull 3P apresentou o melhor desempenho, sendo, portanto, recomendada para a estimar o número de árvores por hectare e por classe diamétrica na região do estudo. Palavras-chave: Manejo florestal; colheita de madeira; modelagem.
\end{abstract}

\begin{abstract}
Pre harvesting modeling of the diametric structure in stands of Pinus spp. using probability density functions. The objective was to study the behavior of the distribution curves diamétric of plantations Pinus spp. in the municipality of Palmas - PR. Data are from a stand of 87 ha, with 18 years of age. The probability functions Normal, Log-normal, Gamma, Beta, Johnson SB, Weibull 2P, 3P Weibull and Weber were adjusted to estimate parameters using the Method of Moments, Method of maximum likelihood and least squares method. The quality of the fits of the distributions was assessed using the $\chi^{2}$ test, with $5 \%$ probability and the relative deviation between the observed and estimated sums of the diameters. There was overlap of the curves in some classes, it was not possible to discriminate the best distribution only observing the histograms. The analytical curves were compared using the chisquare $\left(\chi^{2}\right)$, which revealed significant differences between the observed and estimated curves. There has been analysis of the relative deviations between the observed and estimated sums of the diameters, only for the functions that were adherent in the $\chi^{2}$ test. Among the functions tested, the Weibull 3P function showed the best performance and was recommended to estimate the number of trees per hectare by diameter class in the study region.

Keywords: Forest management; timber harvesting; modeling.
\end{abstract}

\section{INTRODUÇÃO}

As empresas florestais têm buscado otimizar suas atividades a fim de maximizar seus lucros e minimizar seus custos mantendo a qualidade, de modo que todas as ferramentas que possibilitem um melhor planejamento das atividades do manejador são úteis e devem ser consideradas. A caracterização e a definição da distribuição diamétrica são de fundamental importância nos estudos de sortimento dos povoamentos em vários momentos de sua existência, sendo esta a ferramenta mais simples e poderosa para caracterizar a estrutura de uma floresta. De um modo geral, o diâmetro se correlaciona muito bem com outras variáveis importantes, como altura, volume, valor, custo de conversão e sortimento. A determinação da distribuição diamétrica, sua relação com o sítio, composição do povoamento, idade e 
densidade são valiosas tanto para fins econômicos como biológicos (BAILEY; DELL, 1973). Por meio da distribuição diamétrica, é possível identificar o potencial de uso presente e futuro de um povoamento florestal. Um sistema de predição presente e futura da produção, baseado em algumas funções de distribuição, é imprescindível para definir antecipadamente estratégias de manejo, pois possibilita prognosticar o crescimento e a produção florestal dos múltiplos produtos da floresta. As funções de densidade de probabilidade permitem estimar a provável distribuição de diâmetros que o povoamento apresentará descrevendo sua estrutura e facilitando o planejamento da produção da floresta. Essas funções, quando associadas às funções de afilamento, permitem a quantificação do sortimento do povoamento em pé e, quando associadas às classes de sortimentos com seus respectivos preços, permitem o cálculo da renda bruta, e ainda, quando associadas a uma função de produção, permitem estimativas da evolução dos sortimentos do povoamento, bem como da evolução de suas rendas (MACHADO et al., 1990).

O uso de modelos biométricos em estudos populacionais é recorrente em diversas situações, seja para a descrição dos seus parâmetros, seja para a avaliação indireta de efeitos sob o ambiente onde a população se encontra ou para prognose, especialmente por meio de simulação. Entre os modelos biométricos, um dos mais frequentes e básicos é a Função de Distribuição de Probabilidade. Existem diversas funções de distribuição de probabilidade para variáveis aleatórias discretas e contínuas. Entre as que se ajustam a dados discretos estão a Bernoulli, a Binomial, a Binomial Negativa, a Hipergeométrica, a Geométrica e a Poisson. Para as distribuições uniformes, as distribuições são: Normal, Log-normal, Gamma, Gumbel, Weibull, Exponencial, Beta, Qui-quadrado e t de Student, entre outras, podendo ser ajustadas a séries de dados amostrais de variáveis aleatórias contínuas (CARGNELUTTI FILHO et al., 2004).

Com o objetivo de determinar as distribuições de probabilidade mais adequadas e fornecer condições para simulações da estrutura de populações de Curatella americana em ambientes de savana aberta no extremo norte amazônico, Mourão Jr. e Barbosa (2005) aplicaram 18 distribuições de probabilidade. Concluíram que, mesmo com um baixo número de indivíduos amostrados, as distribuições de probabilidade de $C$. americana nesses ambientes seguem os padrões log-normal, para diâmetro de copa e diâmetro de base, e normal, para altura total. Vários outros trabalhos com o objetivo de identificar a distribuição de probabilidade de variáveis aleatórias foram realizados em várias áreas da pesquisa científica. Na área florestal, a adequação das funções de densidade probabilísticas Normal, Log-normal, Beta, Gamma, Weibull e Sb de Johnson foram estudadas por Bartoszeck et al. (2004), Finger (1982), Thiersch (1997), Scolforo e Machado (1996), Abreu et al. (2002), Couto (1980) e Cunha (1995). Contudo, segundo Arce (2004), não existem ainda referências claras sobre os critérios de aplicabilidade das diversas funções de distribuição probabilística nos trabalhos florestais, sendo difícil encontrar alguma evidência biológica sugerindo uma distribuição específica.

Embora muitas pesquisas tenham comprovado a eficiência e a superioridade de algumas funções de distribuição para algumas situações, nunca haverá uma ideal para todos os casos, sendo recomendado o ajuste para cada situação. Prova disso tem-se ao analisar a grande variedade de resultados obtidos pelos pesquisadores. Ressalte-se que os resultados obtidos com as diferentes distribuições probabilísticas variam de acordo com o método utilizado para estimar os parâmetros e com o critério empregado para sua comparação (MALTAMO et al., 1995).

Para estimar os parâmetros dessas distribuições, foram desenvolvidos diferentes métodos de ajuste. Três métodos podem ser destacados: o Método da Máxima Verossimilhança, o Método dos Momentos e o Método dos Percentis (SCOLFORO, 1998). Em teoria, esses métodos deveriam apresentar resultados semelhantes na modelagem da distribuição diamétrica, no entanto, podem ocorrer distorções que tornam as distribuições estimadas por eles estatisticamente diferentes. Embora, em princípio, possam ser utilizados todos os métodos para as estimativas dos parâmetros de qualquer distribuição, as pesquisas têm conduzido a métodos específicos para cada distribuição. Um caso específico é uso do método de regressão linear múltipla (para a distribuição beta). Machado et al. (2000) citam a importância de diversos autores realizarem pesquisas sobre funções de densidade de probabilidade, para representar a distribuição de frequências, por unidade de área para diversas formações florestais e sistemas de manejo, tanto em plantios como em florestas nativas.

Ao se ajustar uma distribuição de probabilidade a um conjunto de dados, trabalha-se com a hipótese de que a distribuição pode representar adequadamente aquele conjunto de informações (CATALUNHA et al., 2002). No presente estudo, trabalhou-se com esse mesmo conceito, e o objetivo foi avaliar comparativamente o comportamento das distribuições probabilísticas Normal, Log-normal, Gamma, Beta, SB de Johnson, Weibull com dois e três parâmetros (Weibull 2P e Weibull 3P) e Weber, sob a hipótese de que uma das funções testadas descreve adequadamente a distribuição de diâmetros dos plantios de Pinus spp. na região centro-sul do estado do Paraná. 


\section{MATERIAL E MÉTODOS}

A área localiza-se no município de Palmas, Paraná, latitude $26^{\circ} 29^{\prime} 03^{\prime \prime}$ Sul e a uma longitude 51 59'26" Oeste, com altitudes variando entre 1035 a 1356 m s.n.m.m. O clima da região de estudo, conforme classificação de Koeppen, é caracterizado como Subtropical Úmido Mesotérmico (Cfb), tendo os verões frescos e os invernos com a ocorrência de severas geadas, não possuindo estações secas. A temperatura média nos meses mais quentes é inferior a $22{ }^{\circ} \mathrm{C}$ e a dos meses mais frios superior a $18{ }^{\circ} \mathrm{C}$. O substrato geológico da região de estudo é formado pelo derrame de Trapp da formação da Serra Geral. Os solos são orgânicos e hidromórficos, com o predomínio de Neossolos Litólicos, Cambissolos e Argissolos (PARANÁ, 1987). O relevo da região varia de plano a ondulado e montanhoso, sendo o último de maior predominância.

Trata-se de uma área de 87 ha com 18 anos de idade onde não foram realizados tratamentos silviculturais e no momento do inventário se encontrava consorciada com bovinos. A amplitude de classe de diâmetro para estudos de crescimento e produção tem sido definida de acordo com o porte das árvores. Assim, encontram-se amplitudes de 1 a $5 \mathrm{~cm}$, para plantios florestais (SCOLFORO; THIERSCHI, 1998; SOARES, 2006; CAMPOS; LEITE, 2009) e amplitudes acima disso para florestas inequiâneas (UMAÑA; ALENCAR, 1998; CUNHA et al., 2002). Tendo em vista que a comercialização da madeira do povoamento em análise será realizada em classes de sortimento com amplitude de $5 \mathrm{~cm}$ e esta ser uma prática constante na pesquisa florestal, conforme trabalhos supracitados, no presente estudo optou-se por adotar o mesmo critério quanto à amplitude das classes.

Para determinar os parâmetros das funções e posterior estimativa do número de árvores, foi utilizado o programa MS EXCEL 2007 e o suplemento Solver. O software possibilitou estimar os parâmetros das distribuições Normal, Log-normal, Beta, Gamma e Weibull 2P e 3P, por meio da iteração de valores paramétricos iniciais, "sementes", pelo Método de Mínimos Quadrados. Os coeficientes das funções Sb de Johnson e Weber foram determinados por meio do emprego do Método dos Momentos. As funções de densidade de probabilidade testadas e suas condicionantes estão apresentadas na tabela 1 .

A simples visualização dos dados amostrais de uma variável em um histograma de frequência não basta para inferir, entre as diversas funções de distribuição de probabilidade conhecidas, a que melhor se ajusta aos dados, além de ser um critério subjetivo. Existem diversos testes não paramétricos, como Kolmogorov-Smirnov, teste de G, $\chi^{2}$, Lilliefors e Shapiro-Wilk, que servem para comparar as probabilidades empíricas de uma variável às probabilidades teóricas estimadas pela função de distribuição (CAMPOS et al., 1983). Na área florestal, os testes de aderência de Kolmogorov-Smirnov e $\chi^{2}$ foram muito difundidos para decidir sobre a função probabilística e sobre o método de ajuste que melhor descreve a estrutura dos povoamentos, no entanto eles não avaliam os erros discriminados por classes diamétricas. No presente trabalho, o critério de decisão adotado na escolha da melhor função foi aderência no teste de $\chi^{2}$, e adicionalmente foram utilizadas as somas de diâmetros observados e estimados expressas de forma relativa.

As somas podem considerar os diâmetros elevados à primeira, segunda, terceira ou quarta potências, segundo queiram ser enfatizados, respectivamente, os desvios diamétricos, em área basal, em volume ou no valor econômico da floresta (MALTAMO et al., 1995). As somas de diâmetros podem ser obtidas também discriminadas por classes diamétricas, para detectar os setores das distribuições nos quais o ajuste das funções apresenta maiores discrepâncias. Esse fato é particularmente interessante, considerando que os erros nas classes diamétricas superiores exercem grande impacto na porção economicamente mais valiosa da floresta (ARCE, 2004). O desvio relativo entre as somas observada e estimada dos diâmetros foi obtido pela equação a seguir:

Em que: $D=$ soma observada dos diâmetros;

$$
\text { Desvio\% }=\frac{D-\hat{D}}{D} * 100
$$

$\hat{D}=$ soma estimada dos diâmetros.

A soma observada dos diâmetros foi obtida diretamente somando-se todos os diâmetros do povoamento elevados à potência desejada. A soma estimada dos diâmetros foi obtida partindo da soma dos produtos entre as frequências estimadas pela função ajustada para classes diamétricas e o diâmetro central de cada classe. Maltamo et al. (1995) reportam que, quando se considera o povoamento inteiro, as somas estimada e observada dos diâmetros podem apresentar valores bastante semelhantes, mesmo que as funções de probabilidade ajustadas não representem bem a realidade. Com a finalidade de detectar os intervalos das distribuições nos quais o ajuste das funções probabilísticas apresenta maiores discrepâncias, foram calculados os desvios relativos para cada classe. 
Tabela 1. Funções de densidade probabilística testadas e suas condicionantes.

Table 1. Probability density functions tested and their restrictions.

Normal

$$
f(x)=\frac{1}{\sigma \sqrt{2 \pi}} e^{-\frac{1}{2}\left(\frac{x-\mu}{\sigma}\right)^{2}}
$$

$$
\sigma>0
$$

$-\infty<x<+\infty$

$-\infty<\mu<+\infty$

Log-normal

$$
f(x)=\frac{1}{\sigma \sqrt{2 \pi}} e^{-\frac{1}{2}\left(\frac{\ln (x)-\mu}{\sigma}\right)^{2}}
$$

$x \geq x^{-} ; \sigma>0$;

$-\infty<x<+\infty$;

$-\infty<\mu<+\infty$

Beta

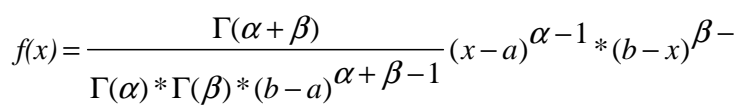

$$
f(x)=\frac{x^{(\alpha-a)} * e^{\frac{-(x-a)}{\beta}}}{\beta^{\alpha} * \Gamma(\alpha)}
$$

Gamma

Sb de Johnson

$$
f(x)=\frac{\delta}{\sqrt{2 \pi}} \frac{\lambda}{(d-\varepsilon)(\lambda+\varepsilon-d)} e^{\left[-\frac{1}{2}\left(\gamma+\delta \ln \left(\frac{x-\varepsilon}{\lambda+\varepsilon-x}\right)\right.\right.} \begin{array}{r}
\varepsilon<x<\lambda ; \\
-\infty<\gamma<+\infty ; \\
\lambda>0 ; \delta>0 .
\end{array}
$$

Weibull 2P

$$
f(x)=\left(\frac{c}{b}\right)\left(\frac{x}{b}\right)^{c-1} \cdot e^{\left[-\left(\frac{x}{c}\right)^{c}\right]} \begin{gathered}
x>0 ; \\
b, c>0 .
\end{gathered}
$$

Weibull 3P

$$
f(x)=\left(\frac{c}{b}\right)\left(\frac{x}{b}\right)^{c-1} \cdot e^{\left[-\left(\frac{x-a}{c}\right)^{c}\right]}
$$

$$
\begin{gathered}
x \geq x_{\text {min }} ; \\
-\infty<x_{\text {min }}<+\infty ; \\
b, c>0 ; \quad a \geq 0 .
\end{gathered}
$$

Weber

$$
f(x)=\frac{x^{a}}{(b+c x)^{d}}
$$

$$
\begin{aligned}
-\infty & <x<+\infty ; \\
d & \geq a+1 ; \\
d & \neq a+2 ; \\
d & \neq a+3 ;
\end{aligned}
$$

$a, b, c, d>0$.

$f(x)$ : função densidade probabilística da variável $x ; x$ : variável aleatória (diâmetro); $\pi$ : constante PI $(3,14151618 \ldots) ; e$ : constante de Euler $(2,7182 \ldots) ; x_{\text {min: }}$ menor valor observado da variável aleatória; $a, b, c, d, \alpha, \beta, \delta, \varepsilon, \gamma, \lambda$ : parâmetros a serem estimados. 


\section{RESULTADOS E DISCUSSÃO}

Foram utilizados 846 indivíduos, com diâmetros entre 15,6 e $46,5 \mathrm{~cm}$, média aritmética de $27,8 \mathrm{~cm}$ e desvio-padrão de 5,33 . O valor mais frequente foi $25 \mathrm{~cm}$ e a mediana foi de $27,3 \mathrm{~cm}$. Os coeficientes de assimetria e curtose são 0,3576 e 0,14213 , respectivamente. Os testes de normalidade de Kolmogorov-Smirnov, Cramer-von Mises e Anderson-Darling indicaram a existência de distribuição normal. $\mathrm{Na}$ avaliação da concentração de árvores por classe de diâmetro, tomaram-se como base as frequências relativas por classe diamétrica observadas. Foi possível observar que $84 \%$ dos indivíduos estão concentrados na classe de diâmetro que vai de 25 a $35 \mathrm{~cm}$.

Na tabela 2 são apresentados os parâmetros estimados para as distribuições probabilísticas. Esses valores foram aplicados nas funções para obterem-se as frequências relativas por classes diamétricas. Arce (2004), ao analisar a estrutura de plantios clonais de Populus deltoides Marsh., utilizou a distribuição normal, no entanto encontrou valores negativos para limite inferior. Esse fato não foi observado neste trabalho, o que indica que, embora se trate de uma função probabilística, não apresenta distorções do ponto de vista biológico. Quando no ajuste dessas funções são apresentados limites negativos, eles indicam valores tendendo a $-\infty$. Essa distorção pode ser minimizada avaliando-se a função quanto ao seu desempenho na porção da distribuição em que há coerência na descrição dos dados.

Tabela 2. Parâmetros estimados das funções de distribuição e estatística $\chi^{2}$.

Table 2. Estimated parameters of distribution functions and $\chi^{2}$ statistica.

\begin{tabular}{llcc}
\hline Nome & & Coeficientes & $\chi^{2}$ \\
\hline \multirow{2}{*}{ Normal } & $\mu$ & 27.8868 & $24.36498 * *$ \\
\hline \multirow{2}{*}{ Log-normal } & $\sigma$ & $\mu .3357$ & 8.059693 \\
\hline \multirow{2}{*}{ Beta } & $\sigma$ & 0.1925 & 13.28254 \\
\hline \multirow{2}{*}{ Gamma } & $\alpha$ & 2.7955 & 10.21471 \\
\hline \multirow{2}{*}{ Sb de Johnson } & $\beta$ & 4.2279 & $16.18164 * *$ \\
\hline \multirow{2}{*}{ Weibull 2P } & $\alpha$ & 27.3162 & $94.81206 * *$ \\
\hline \multirow{2}{*}{ Weibull 3P } & $\beta$ & 1.0209 & \multirow{2}{*}{0.828868} \\
& $\delta$ & 1,187801 & 20,126104 \\
\hline \multirow{2}{*}{ Weber } & $\gamma$ & 29,70689 & 5,759766 \\
\end{tabular}

$\chi_{(0,05 ; 0,01)}^{2}-14,06714 ; 18,47531 ; * *$ significativo ao nível de $1 \%$ de probabilidade de erro.

Como se pode observar na tabela 2 , os valores do teste de $\chi^{2}$ que foram menores que o tabelado indicam a não rejeição da hipótese de nulidade, concluindo-se que as frequências esperadas e observadas são similares sob o ponto de vista estatístico. Em contrapartida, os ajustes que apresentaram valores significativos são inadequados para descrever o conjunto de dados. De acordo com o teste de $\chi^{2}$, as funções que se demonstraram aderentes foram Log-normal, Beta, Gamma e Weibull 3P; as funções Normal, Sb de Johnson, Weibull 2P e Weber não foram aderentes tanto ao nível de 1 quanto $5 \%$ de probabilidade de erro no teste bilateral. As estimativas foram analisadas por classe diamétrica, na tentativa de evidenciar os setores da distribuição em que as funções foram mais imprecisas na predição do número de diâmetros. A função Normal apresentou grandes discrepâncias no ajuste das classes inferiores, chegando a diferenças de até $50 \%$ nas classes de 20 a $30 \mathrm{~cm}$. A função Log-normal só não modelou de 
maneira satisfatória a classe central, onde apresentou seperestimativas da ordem de 30\%, o mesmo ocorrendo para a função Gamma. A classe em que a função Beta apresentou as maiores discrepâncias foi de 35 a $40 \mathrm{~cm}$, com superestimativas da ordem de 50\%. As maiores diferenças nas estimativas da função $\mathrm{Sb}$ de Johnson estão nas classes centrais e superiores, chegando a $80 \%$. Para as funções Weibull $2 \mathrm{P}$ e Weber, as discrepâncias chegam a $100 \%$ ao longo de toda a distribuição. A função Weibull 3P foi a que apresentou as menores discrepâncias por classe diamétrica, sendo que a classe central é onde se verificam os maiores erros. No entanto, como a probabilidade nessa classe é elevada, entende-se que erros de pequena ordem nessa classe são de menor importância, pois não prejudicam a forma da distribuição.

Os resultados corroboram estudos anteriores, como Maltamo et al. (1995), que reportam a distribuição Weibull como superior à função Beta para Pinus taeda e superior a distribuições Sb de Johnson para Pinus patula. Schneider et al. (2008), modelando a estrutura de Pinus taeda, utilizaram regressão aninhada para estimar os parâmetros da função de Weibull e também reportam resultados satisfatórios para essa função. Ressalte-se que resultados diferentes podem ser obtidos com as distribuições probabilísticas dependendo do método utilizado para estimar os parâmetros e do critério empregado na comparação.

O desempenho das funções está expresso graficamente na figura 1, permitindo uma melhor visualização das variações na distribuição, bem como das frequências observadas e estimadas por classe diamétrica para cada função.

Observa-se nos gráficos a dificuldade na modelagem do número de árvores nas menores e nas maiores classes. As funções que reportam resultados satisfatórios para as classes menores são $\mathrm{Sb}$ de Johnson, Beta e Gamma, sendo que esta última superestima o número de árvores nas classes centrais e as outras duas apresentam subestimativas nessas classes. No entanto, as três apresentam boas estimativas para as classes inferiores. A função Weibull 3P apresenta estimativas satisfatórias tanto para as classes inferiores quanto para as superiores. A análise do desempenho das funções em histogramas, apesar de válida, é subjetiva, o que não confere a esse método total confiabilidade, motivo pelo qual, no presente estudo, adicionalmente optou-se por analisar graficamente os desvios relativos das somas de diâmetros elevados à primeira potência por classes diamétricas somente para as distribuições aderentes no teste de $\chi^{2}$ (Figura 2).

Os desvios relativos entre as somas observadas e estimadas de diâmetros elevados à primeira potência foram suficientes para avaliar o desempenho das funções. Apesar de a diferença entre as funções não ser grande, comprova a superioridade do modelo Weibull 3P. Os valores mais elevados na análise desse critério costumam estar presentes apenas nas idades iniciais dos plantios, independentemente da função analisada e do método empregado para a estimativa dos coeficientes. Arce (2004) usou esse critério para analisar o desempenho de funções na modelagem da estrutura de plantios clonais de Populus e encontrou desvios de 11 a $13 \%$ para as idades iniciais, sendo que no restante das idades os desvios observados, para desvios elevados à quarta potência, que reflete melhor a caracterização econômica da floresta, não superaram o valor de $0,005(0,5 \%)$.

No presente estudo, devido à grande heterogeneidade das condições do plantio, conforme se visualiza na figura anterior, os desvios chegam a $100 \%$ para a função Beta, nas classes inferiores e superiores. Essa função, dentre as funções aderentes no teste de $\chi^{2}$, foi a que apresentou os piores resultados. A função Log-normal apresenta desempenho semelhante nas classes inferiores a superiores, onde reporta os piores resultados, sendo que nas classes intermediárias também não apresenta resultados satisfatórios. A julgar por esse critério, a função Gamma é a que reporta melhores resultados, pois apresenta desvios mais homogêneos ao longo das classes diamétricas, mas superestima o número de diâmetros na maioria das classes. A função Weibull 3P, apesar de não apresentar os melhores resultados em uma análise gráfica preliminar, foi a que apresentou a menor soma dos desvios relativos entre as frequências observadas e estimadas. Na Figura 3, está apresentada a distribuição diamétrica observada e a distribuição estimada pela função Weibull 3P. Essa função mostra-se flexível, apresentando resultados satisfatórios.

Este trabalho foi desenvolvido com dados tomados em apenas uma observação, no entanto, devese lembrar que, para a construção de um modelo genérico de predição de parâmetros, deve-se dispor de dados distribuídos ao longo de toda a amplitude de idades, densidades e sítios. Portanto, os resultados apresentados neste trabalho não devem ser considerados como definitivos, porém contribuem de forma substancial para pesquisas conduzidas nessa linha, tendo em vista que a maior parte dos trabalhos foi feita com plantios que receberam algum tipo de tratamento silvicultural. 

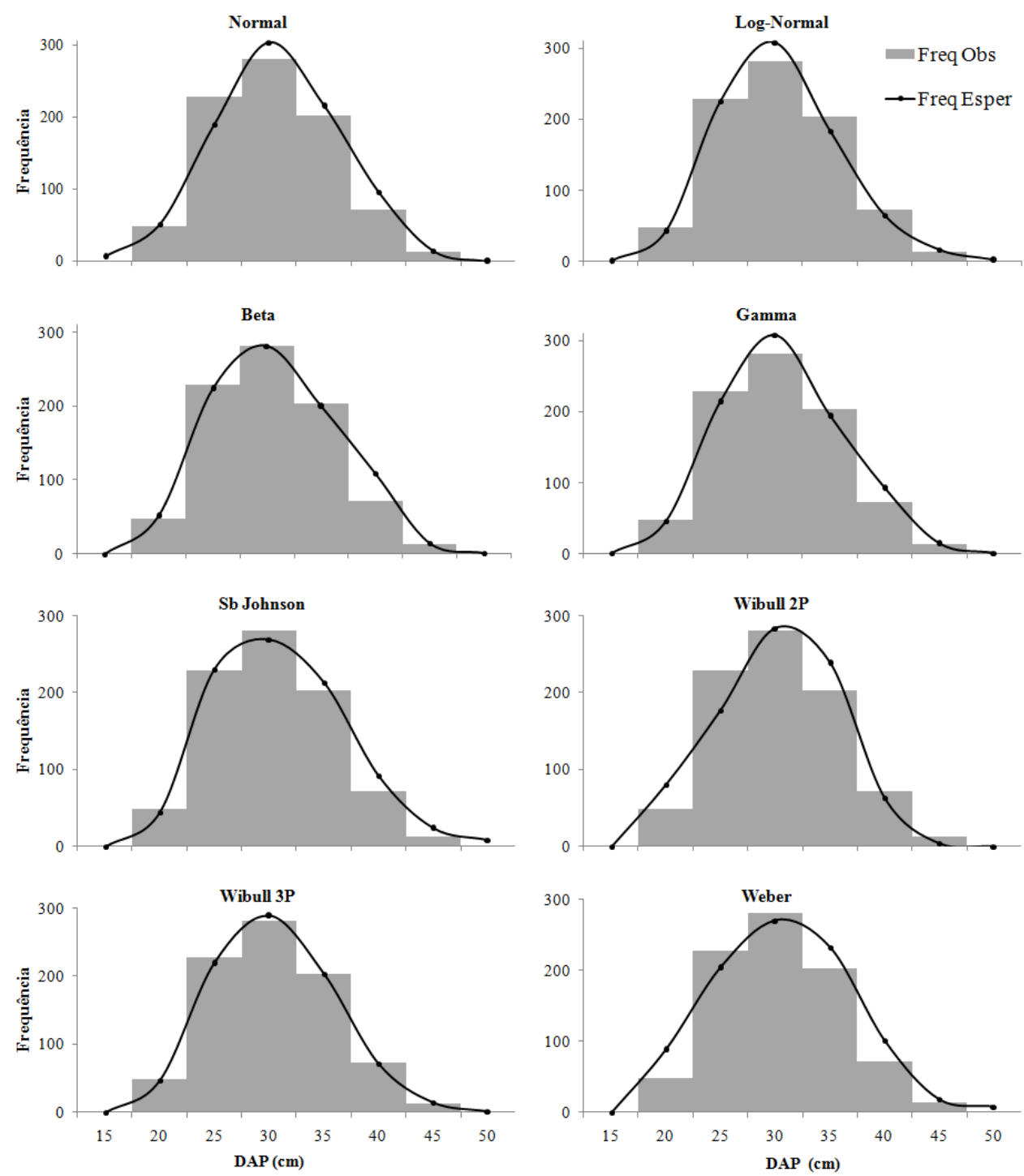

Figura 1. Curvas de ajuste dos oito modelos probabilísticos testados sobre a distribuição de frequências observadas para a variável DAP.

Figure 1. Tuning curves of the eight probabilistic distribution models tested on the distribution of observed frequencies for the variable DBH.

O modelo Weibull 3P, ajustado pelo método de mínimos quadrados, apresentou distribuição de resíduos satisfatória, com baixos desvios por classe de diâmetro. Os demais modelos, apesar de apresentarem boas estimativas do número total de árvores, mostraram-se demasiadamente tendenciosos e imprecisos nas classes inferiores e superiores.

Tendo em vista que só se dispunha de dados de uma observação e não da evolução dos plantios em diferentes idades, seria precipitado comparar e concluir qual método de ajuste para a função Weibull seria mais indicado para plantios na região estudada. Porém, como se necessitava de um valor inicial para o coeficiente $c$ para poder gerar o processo iterativo no método da máxima verossimilhança, optou-se por obtê-lo iterativamente. Estudos dedicados a analisar o comportamento das distribuições onde diferentes métodos são empregados para estimativa dos parâmetros das funções são de grande importância, considerando que algumas pesquisas comprovaram a sensibilidade das funções aos métodos utilizados. 

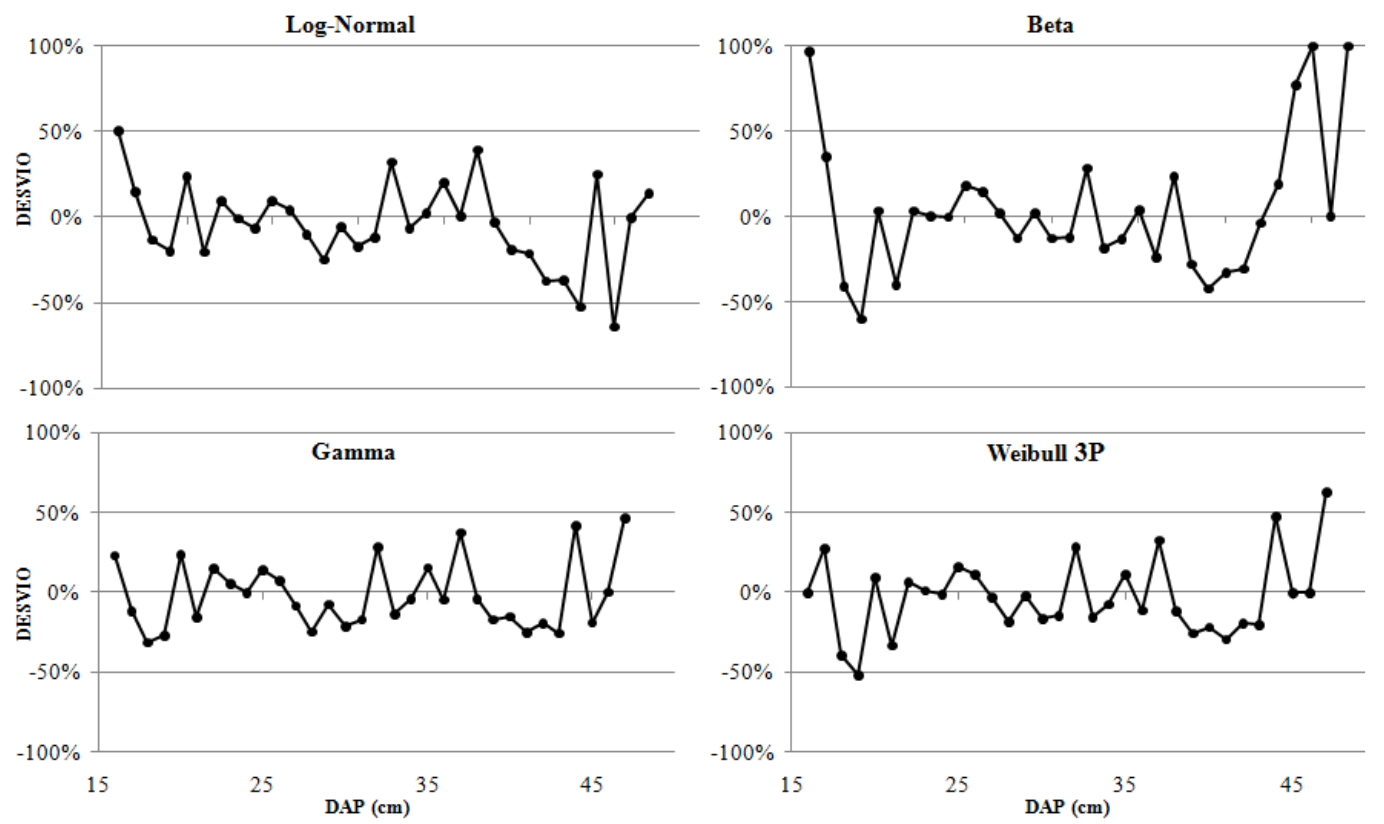

Figura 2. Desvios relativos das somas de diâmetros elevados à primeira potência por classes diamétricas para as quatro distribuições que se mostraram aderentes no teste de $\chi^{2}$.

Figure 2. Relative deviations of sums of diameters raised to the first power by diameter classes for the four distributions that were shown to be members of the $\chi^{2}$ test.

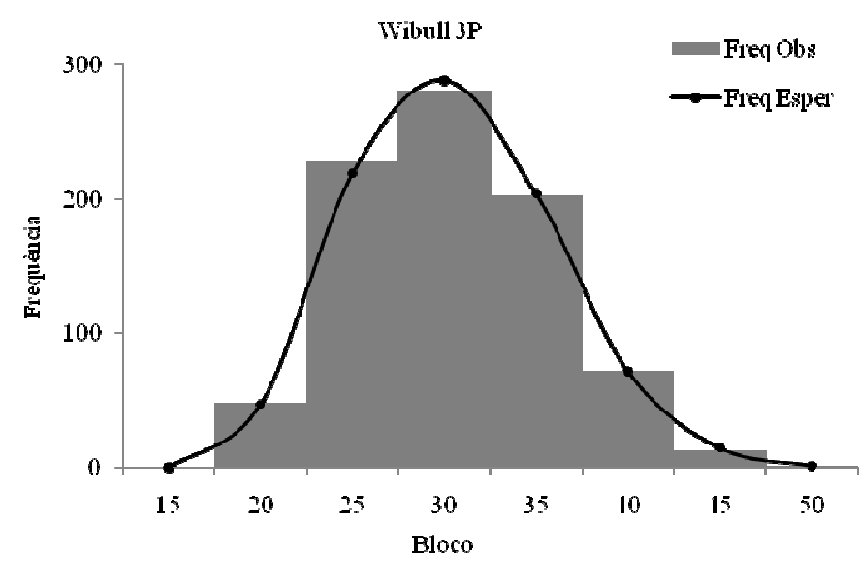

Figura 3. Distribuição diamétrica observada e a distribuição estimada pela função Weibull 3P.

Figure 3. Diameter distribution observed and estimated to the Weibull 3P distribution function.

Em estudos sobre distribuições diamétricas e no uso delas como ferramenta na predição dos sortimentos, podem ser utilizadas distribuições ajustadas tanto por um quanto por outro método. A decisão e responsabilidade no uso de cada método é inteiramente do profissional que o faz, apesar da literatura fazer indicações de métodos para cada função. Contudo, no presente estudo não se optou por trabalhar com as distribuições ajustadas por um único método. Atualmente, muitas linhas de pesquisa estão atuando nessa área com a finalidade de desenvolver modelos de crescimento e produção baseados na distribuição diamétrica, contemplando não somente plantios experimentais como também comerciais. A aplicação dessas técnicas a dados provenientes de plantios comerciais, localizados de modo a abranger uma amplitude de classes de sítio, idade e ainda opções de manejo, tais como densidades de plantio, 
desbastes e conduções, entre outros critérios, são etapas seguintes a serem realizadas. A simulação contínua do manejo dos povoamentos apoiada por critérios de decisão técnica e econômica é de extrema importância, pois permite a otimização da produção florestal.

\section{CONCLUSÕES}

- A função de densidade de probabilidade Weibull demonstrou boa capacidade para representar o conjunto de dados. Essa função ajustada com três parâmetros apresentou os melhores resultados, propiciando estimativas precisas da distribuição diamétrica de plantios de Pinus spp. no centro-sul do estado do Paraná.

- Recomenda-se testar outros métodos de ajuste para esta e outras funções, bem como sua sensibilidade aos diversos atributos dos povoamentos. Selecionar os atributos mais correlacionados com os parâmetros das funções e estimá-los na idade desejada permitirá conhecer a nova estrutura da floresta, possibilitando prognosticar a produção em outras situações, como diferentes idades, sítios e sistemas de manejo.

\section{REFERÊNCIAS}

ABREU, E. C. R.; SCOLfORO, J. R. S.; OlIVEIRA, A. D.; MELlO, J. M.; KANEGAE, H. J. Modelagem para prognose precoce do volume por classe diamétrica para Eucalyptus grandis. Revista Scientia Forestalis, Piracicaba, n. 61, p. 86 - 102, 2002.

ARCE, J. E. Modelagem da estrutura de florestas clonais de Populus deltoides March através de distribuições diamétricas probabilísticas. Ciência Florestal, Santa Maria, v. 14, n. 1, p. 149 - 164, 2004.

BAILEY, R. L.; DELL, T. R. Quantifying diameter distributions with the Weibull function. For. Sci., v. 19, p. 97 - 104, 1973.

BARTOSZECK, A.; MACHADO, S. do A.; FIGUEIREDO FILHO, A.; OLIVEIRA, E. B. A distribuição diamétrica para bracatingais em diferentes idades, sítios e densidades na Região Metropolitana de Curitiba. Floresta, Curitiba, v. 34, n. 3, p. 305 - 323, 2004.

CAMPOS, J. C. C.; RIBEIRO, J. C.; COUTO, L. Emprego da distribuição diamétrica na determinação da intensidade de corte em matas naturais submetidas ao sistema de seleção. Revista Árvore, Viçosa, v. 7, n. 2, p. $110-122,1983$.

CAMPOS, J. C. C.; LEITE, H. G. Mensuração florestal: perguntas e respostas. Viçosa: UFV, 2009. v. 3. $548 \mathrm{p}$.

CARGNELUTTI FILHO, A.; MATZENAUER, R.; TRINDADE, J. K. Ajustes de funções de distribuição de probabilidade à radiação solar global no estado do Rio Grande do Sul. Pesquisa Agropecuária Brasileira, Brasília, v. 39 , n. 12, p. 1157 - 1166, 2004.

CATAlunhA, M. J.; SEDIYAMA, G. C.; LEAL, B. G. Aplicação de cinco funções densidade de probabilidade a séries de precipitação pluvial no Estado de Minas Gerais. Revista Brasileira de Agrometeorologia, Santa Maria, v. 10, n. 1, p. 153 - 162, 2002.

COUTO, H. T. Z. Distribuições de diâmetros em plantações de Pinus caribaea Morelet var. caribaea. 83 f. Tese (Doutorado em Ciências Florestais) - Escola Superior de Agricultura "Luiz de Queiroz", Piracicaba, 1980.

CUNHA, U. S. Análise da estrutura diamétrica de uma floresta tropical úmida da Amazônia Brasileira. 134 f. Dissertação (Mestrado em Ciências Florestais) - Universidade Federal do Paraná, Curitiba, 1995.

CUNHA, U. S. da; MACHADO, S. do A.; FIGUEIREDO FILHO, A.; SANQUETTA, C. R. Predição da estrutura diamétrica de espécies comerciais de terra firme da Amazônia por meio de matriz de transição.

Ciência Florestal, v. 12, p. 109 - 122, 2002. 
FINGER, C. A. G. Distribuição de diâmetros em acácia-negra, Acacia mearnsii de Wild em diferentes povoamentos e idades. $124 \mathrm{f}$. Dissertação (Mestrado em Ciências Florestais) - Universidade Federal do Paraná, Curitiba, 1982.

MACHADO, S. A.; MELLO, J. M.; BARROS, A. B. Comparação entre métodos para avaliação de volume total de madeira por unidade de área, para o pinheiro-do-paraná, na região sul do Brasil. Cerne, v. 6, n. 2, p. 55 - 66, 2000.

MACHADO, S. A; MARQUESINI, L. C. P. T.; BACOVIS, R. Effects of age, site, average DBH and density upon the diameter distribution of thinned products from Pinus taeda plantations in the State of Paraná, Brazil. XIX World Congress; In: IUFRO. Montreal, Canadá. 1990.

MALTAMO, M.; PUUMALAINEN, J.; PÄIVINEN, R. Comparison of Beta and Weibull Functions for Modelling Basal Area Diameter Distribution in Stands of Pinus sylvestris and Picea abies. Scand. J. For. Res., v. 10, p. 284 - 295, 1995.

MOURÃO Jr., M.; BARBOSA, R. I. Distribuições de probabilidade de parâmetros dendrométricos. Embrapa Bioecologia do Caimbé [Curatella americana L. (Dilleniaceae)] (i): Boa Vista, 2005. (Comunicado Técnico, n. 18).

PARANÁ. Secretaria e Estado da Agricultura e Abastecimento, Instituto de Terras, Cartografia e Florestas. Atlas do Estado do Paraná, Curitiba, 1987. 73 p.

SCOLFORO, J. R. S.; MACHADO, S. do A. Um sistema de crescimento e produção com simulador de desbaste. Revista Scientia Forestalis, Piracicaba, n. 50, p. 51 - 64, 1996.

SCOLFORO, J. R. S. Manejo Florestal. Lavras: Universidade Federal de Lavras. Fundação de Apoio ao Ensino, Pesquisa e Extensão, 1998. 310 p.

SCOLFORO, J. R. S.; THIERSCHI, A. Estimativas e testes da distribuição de frequência diamétrica para Eucalyptus camaldulensis, através da distribuição $\mathrm{Sb}$, por diferentes métodos de ajuste. Scientia Forestalis, n. 54, p. 93 - 106, 1998.

SCHNEIDER, P. R.; FINGER, C. A. G.; BERNETT, L. G.; SCHNEIDER, S. P.; FLEIG, F. D. Estimativa dos parâmetros da função de densidade probabilística de Weibull por regressão aninhada em povoamento desbastado de Pinus taeda L. Ciência Florestal, Santa Maria, v. 18, n. 3/4, p. 375 - 385, 2008.

SOARES, T. S. Avaliação de modelos de distribuição diamétrica em povoamentos de eucalipto não desbastados. 2006. 69 p. Tese (Doutorado) - Universidade Federal de Viçosa, Viçosa.

THIERSCH, A. Eficiência das distribuições diamétricas para prognose da produção de Eucalyptus camaldulensis. 1997. 115 p. Dissertação (Mestrado em Ciências Florestais) - Universidade Federal de Lavras, Lavras.

UMAÑA, C. L. A.; ALENCAR, J. C. Distribuições diamétricas da floresta tropical úmida em uma área no Município de Itacoatiara - AM. Acta Amazonica, v. 28, p. 167 - 190, 1998. 